МОДЕЛИРОВАНИЕ ВЕТРОВОГО ВОЛНЕНИЯ И ШИРИНЫ ЗАТОПЛЕНИЯ ПЛЯЖА ДЛЯ ИССЛЕДОВАНИЙ ПРИБРЕЖНЫХ ДЮН НАЦИОНАЛЬНОГО ПАРКА «КУРШСКАЯ КОСА»

\author{
1,2Данченков А.P.
}

${ }^{1}$ Институт океанологии им. П.П. Ширшова РАН, Москва, e-mail: aldanchenkov@mail.ru; ${ }^{2}$ Балтийский федеральный университет имени Иммануила Канта, Калининград

Рассматриваемая в работе геоморфологическая компонента геоэкологической береговой системы Куршской косы, в морфосистемном понимании, является частью береговой литодинамической системы. В условиях особого характера природопользования на территории национального парка «Куршская коса» социального и культурного значения высока геоэкологическая роль дюнного вала, выступающего как первая линия защиты внутренних территорий от штормовых воздействий и эолового ветропесчаного потока. Песчаные пляжи, литодинамически связанные с вдольбереговой литодинамической системой, являются отправной точкой естественного восстановления дюнного вала. Гидродинамические процессы воздействуют на морфодинамическую систему пляжа и дюнного вала, что обуславливает значимость их изучения в исследовании всей береговой геоэкологической системы, в части динамизма протекающих на ней факторов природного происхождения. Гидродинамическим режимом и геоморфологическим строением определяется как наличие наносов на пляже, так и ширина незатопленной части пляжа, являющаяся лимитирующим фактором развития дюнного вала. Ширина незатопленной части пляжа зависит от ширины пляжа, его уклона, уровня моря и высоты волнового наката. При возможности измерений большей части параметров, наблюдения за высотой волнового наката в долгосрочном периоде являются технически и экономически затратной процедурой, что обуславливает использование моделей. В работе использовалась регионально адаптированная модель волнового наката, а также модель, рекомендованная национальным стандартом. Для получения гидродинамических условий в акватории Балтийского моря было выполнено численное моделирование с использованием модели SWAN, а также сравнение результатов с натурными наблюдениями и региональным анализом. Была отмечена приемлемая сходимость с данными натурных наблюдений. Для исследования дюнного вала Национального парка «Куршская коса» хорошо соотносились сведения о ширине пляжа, полученные с использованием региональной адаптации, в сравнении с измеренными значениями. В моделировании ширины затопления пляжей в условиях различных морей рекомендуется использовать региональные адаптации моделей волнового наката.

Ключевые слова: ветровое волнение, прибрежные дюны, песчаные пляжи, моделирование, волновой накат

\title{
WIND WAVES AND BEACH INUNDATION WIDTH MODELLING FOR STUDYING CURONIAN SPIT NATIONAL PARK FOREDUNE STABILITY
}

\section{${ }^{1,2}$ Danchenkov A.R.}

${ }^{1}$ Shirshov Institute of Oceanology, Russian Academy of Sciences, Moscow, e-mail: aldanchenkov@mail.ru; ${ }^{2}$ Immanuel Kant Baltic Federal University, Kaliningrad

The geomorphological component of the geoecological coastal system of the Curonian Spit considered in the work, in the morphosystem understanding, is part of the coastal lithodynamic system. Due to the special nature of nature management in the territory of the Curonian Spit National Park, social and cultural significance, the geoecological role of the dune rampart is high. Since it acts as the first line of defense of inland from storms and aeolian wind and sand flow. Sandy beaches lithodynamically associated with the coastal lithodynamic system are the starting point for the natural restoration of the foredune. Hydrodynamic and processes affect the morphodynamic system of the beach and foredune. This determines the significance of their research in the study of the entire coastal geoecological system, in terms of the dynamism of factors of natural origin occurring on it. The hydrodynamic regime and geomorphological structure determines both the presence of sediments on the beach and the width of the uninundated part of the beach, which is a limiting factor in the development of the foredune. The width of the uninundated part of the beach depends on the width of the beach, its slope, sea level and the height of the wave run-up. If it is possible to measure most of the parameters, monitoring the wave run-up height in the long term is technically and economically costly, which leads to the use of models. In the work, a regionally adapted model of the wave run-up was used, as well as a model recommended by the national standard. To obtain hydrodynamic conditions in the Baltic Sea, a numerical simulation was performed using the SWAN model, as well as comparing the results with field observations and regional analysis. Acceptable convergence with field observations was noted. To study the Curonian Spit National Park foredune, the information about the width of the beach obtained using regional adaptation in comparison with the measured values was well correlated. In modeling the beach inundation width in different seas using regional adaptations of wave run-up models is recommended.

Keywords: wind waves, coastal dunes, sandy beaches, numerical modelling, wave run-up

Из определения прибрежных дюн - форм рельефа, сформировавшихся посредством переноса и переотложения песков у песчаных пляжей, состояние пляжей для устойчивости дюн играет важнейшую роль [1].
Развитие дюны происходит у пляжей при синхронизации транспортирующего потенциала (силы и направления ветра) с наносами, доступными на пляже или поступающими при воздействии гидродинамических 
процессов. Доступность наносов для эолового перемещения связано с понятием полосы насыщения ветропесчаного потока [2]. В формировании полосы насыщения - ширина незатопленной части пляжа является важнейшим параметром, наряду со скоростью и направлением ветра [3; 4]. Ширина затопления пляжа зависит от прибрежных гидродинамических условий (высоты и длины волнения) и высоты нагона моря, соответственно может сокращать доступность наносов для эолового перемещения либо быть фактором абразии прибрежных дюн.

В районах, где не ведется регулярных наблюдений за прибрежным волнением, к которому относится и исследуемый в настоящей работе район (морской аккумулятивный берег Куршской косы), возможно выполнить математическое моделирование для получения параметров ветрового волнения в акватории с целью определения ширины затопления пляжа. Для целей работы было выполнено численное моделирование ветрового волнения с использованием волновой спектральной модели третьего поколения SWAN версии 41.20A [5]. Модель создана для расчета параметров ветрового волнения в прибрежной зоне исходя из рельефа дна и скорости ветра. Модель SWAN для расчета параметров ветрового волнения в прибрежной зоне моря широко используется в мире и доказала свою эффективность [6-8]. С использованием данной модели в юго-восточной части Балтийского моря ранее производились расчеты различных параметров ветрового волнения [9-11]. Модель была верифицирована для использования в Балтийском море [11-13], в том числе в прибрежной зоне.

Для использования в условиях мелководья в модели SWAN реализованы трехкомпонентные схемы взаимодействия волн и две схемы, отвечающие за трение о дно и обрушение волн или диссипацию. При расчете учитываются следующие процессы: распространение волн в пространстве, рефракция (дно и течения), дифракция и др. Среди процессов диссипации и генерации: ветровая генерация, нелинейное взаимодействие (триады), диссипации из-за: обрушения, обрушения, связанного с изменением глубин, диссипация из-за трения о дно.

Цель исследования состояла в применении модельного подхода для определения ширины затопления пляжа на морском аккумулятивном берегу в задачах научно обоснованного управления прибрежной зоной и дюнным валом Куршской косы.

\section{Материалы и методы исследования}

Исходные гидрометеорологические данные

Поскольку пространственные размеры исследуемого района находятся в пределах $16 \times 27$ км, а в непосредственной близости в отрытом море находится действующая метеостанция, в качестве входных данных модели используются сила и направление ветра, полученные с МЛСП Д-6. Результаты реанализа метеополей (например, NCEP/ CFSR), как это используется в ряде работ [11-15], покрывают исследуемый район лишь двумя ячейками, а СКО (среднеквадратическая ошибка), в сравнении с метеопостами, составляет 3,43-1,33 м/с [16]. Дискретность данных по силе и направлению ветра составляла 3 часа. От пунктов наблюдения за шириной затопления пляжа (14 и 42 км Куршской косы) станция удалена на 26.6 и 15.8 км соответственно. Данные по уровню Балтийского моря получены с поста 86179 Пионерский (Калининградский ЦГМС). Дискретность данных уровня моря составляет 6 часов, а «0» поста установлен на отметке $500 \mathrm{~cm}$.

\section{Исходная батиметрическая основа и расчетная сетка модели $S W A N$}

Цифровая модель рельефа дна была построена с использованием ESRI ArcGIS 10.0 по объединенным батиметрическим данным из различных источников: сечением 5 м для глубин более 50 м [17], сечением 1 м, построенным по морским планам ГУНиО, сечением 0,1-0,2 м до глубин 2 м (на основе собственных измерений). Батиметрические данные были объединены (рис. 1, а) с использованием инструмента Merge, на основе чего была построена цифровая модель дна на регулярной сетке (размер ячейки 5 м).

Для исследуемой области была построена нерегулярная расчетная сетка ADCIRC, с. Конечно элементная сетка выполнена триангуляцией, которая хорошо аппроксимирует кривую береговой линии, поскольку количество узлов рассчитывается исходя из количества точек на береговой линии. В областях интереса (полигоны наблюдения за шириной затопления пляжа, платформа Д6) расчетная сетка сгущена для лучшего отображения рельефа дна, в особенности вблизи береговой линии. Вблизи полигонов, а также у платформы Д6 шаг сетки составляет 20-100 м, в глубоководной части сетка разряжена до шага 5 км. Радиус окружности расчетной сетки выбран таким образом, чтобы длина разгона ветровых волн могла составлять не ме- 
нее 150 км. Всего расчетная сетка состоит из 73200 расчетных узлов.

Параметры моделирования: координаты прямоугольные, зональные WGS84 UTM34N, режим GEN3, со следующими параметрами: экспоненциальная схема роста Komen [18] с включенным правилом Agrow. Донное трение принято Friction Jonswap Constant cf 0.071. Включены процессы: забурунивание; трехкомпонентное волновое взаимодействие, разрушение волн. Выходные данные в модели общего расчета формируются в виде файла граничных условий и волнового спектра, а также таблицы с основным и дополнительными параметрами волнения: время (Т); высота значительных волн (Hs), м; длина волны (Wlen), среднее направление волны (WDir), гр., и др. Моделирование с использованием волновой модели SWAN выполнялось для пятилетнего периода 2014-2019 гг., с шагом 3 часа.

\section{Моделирование высоты волнового наката и ширины затопления пляжа}

Расчет высоты волнового наката был выполнен согласно приложению Д, СП 38.13330.2018 [19], применяемого в проектировании берегозащитных сооружений в России, а также по адаптированной для юго-восточной Балтики модели волнового наката [20] (уравнение 1):

$$
R=1,29 H_{s}\left(\tan \beta / \sqrt{\frac{H_{s}}{L}}\right)^{0.72},
$$

где $H_{s}$ - высота значительных волн, м; $L-$ длина волны, м; $\beta$ - уклон пляжа.

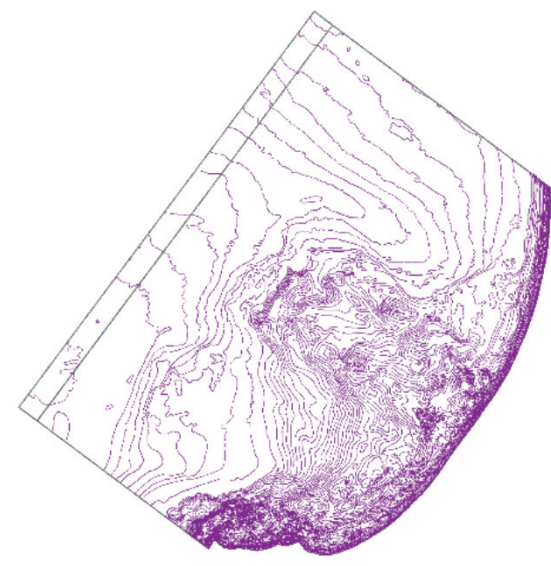

a)
По результатам полученной высоты волнового наката был выполнен расчет ширины затопления пляжа (BI) (уравнение 2):

$$
B I=(R+S) * \operatorname{ctg}(\beta),
$$

где $R$ - высота волнового наката, полученная посредством расчетов по СП 38.13330.2018 или адаптированной модели; $S$ - высота уровня моря, м; $\beta$ - уклон пляжа.

Уклон пляжа определялся по данным мониторинга за состоянием дюнного вала Куршской косы, выполнявшегося с 2014 г., в рамках которого выполнялись наблюдения за шириной затопления пляжа in situ [4;21].

\section{Результаты исследования и их обсуждение}

\section{Моделирование ветрового волнения}

Первым этапом выполняемого исследования являлось сравнение результатов выполненного моделирования ветрового волнения с использованием волновой модели SWAN (далее - SWAN) с данными по ветровому волнению из общедоступных источников (E.U. Copernicus Marine Service (далее - Copernicus) - использующих волновую модель WAM 4.6), а также измерений с использованием уровнемера-волнографа, установленного на морской платформе Д6 (рис. 1). Сравнение и сопоставление результатов проводилось для результатов 2019 г., с шагом 3 часа. Всего использовалось 2375 пар сравнения наблюдений и модельных значений (рис. 2).

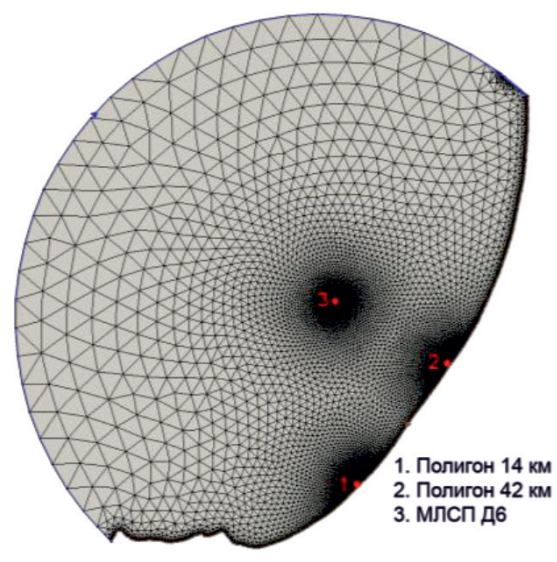

б)

Рис. 1. Цифровая модель рельефа дна: а) исходные батиметрические данные для построения модели дна; б) нерегулярная расчетная сетка ADCIRC 


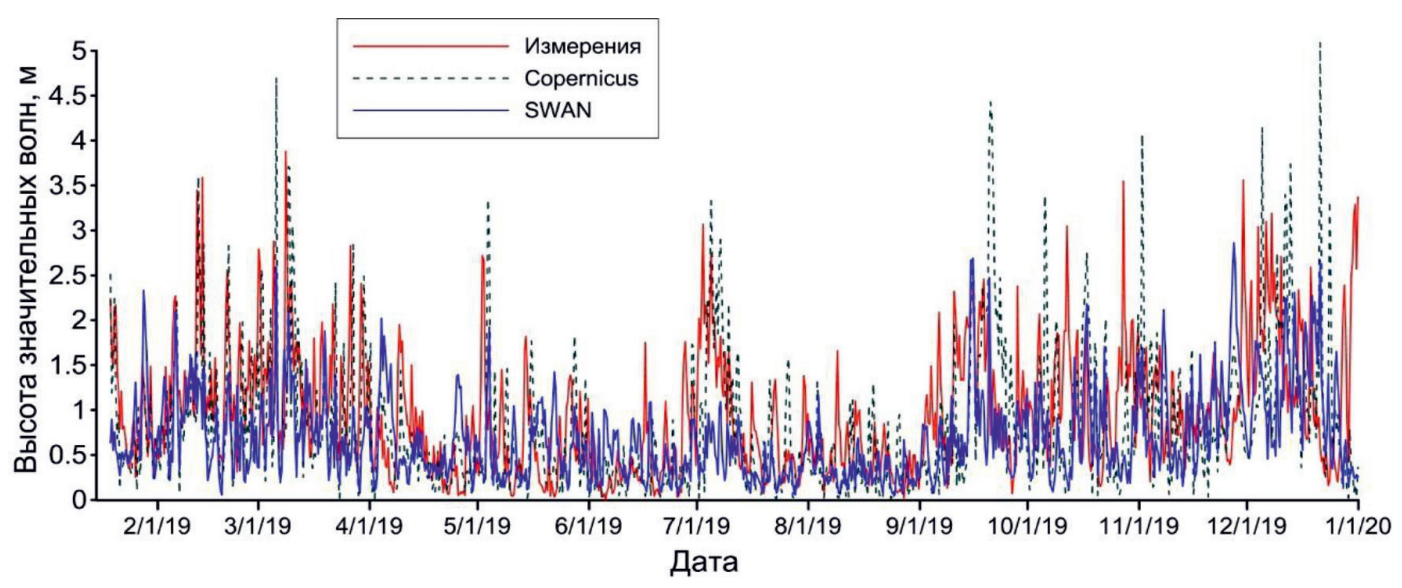

Рис. 2. Значительная высота волн у платформы Д6 в 2019 г. по данным измерений, материалам Cоретпісиs и выполненного моделирования SWAN

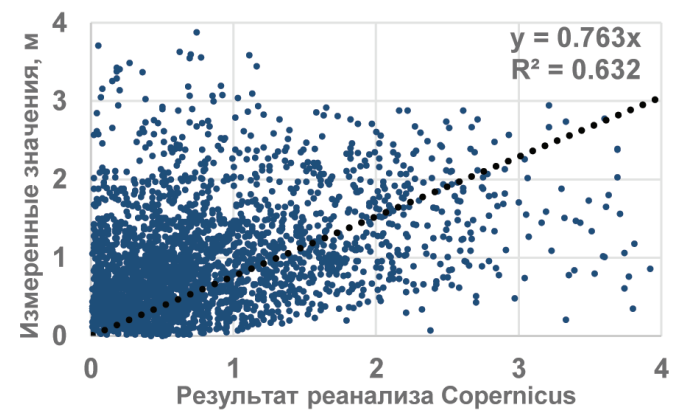

a)

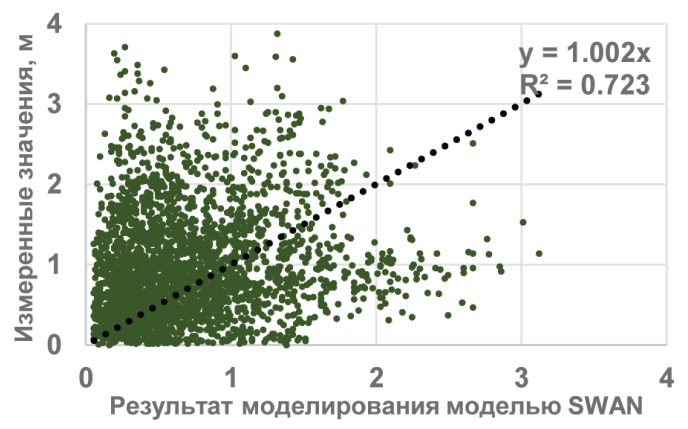

б)

Рис. 3. Скаттер диаграммы измеренных и моделированных значений: а) Copernicus и измеренные значения; б) SWAN и измеренные значения

По графику годового хода значительной высоты волн наблюдаемая разница между полученными с использованием SWAN высотами значительных волн и полученная по результатам прямых измерений, вероятно, связана с дискретностью используемых метеорологических данных. Моделированные значения имеют более плавный ход, в отличие от наблюдаемых, что свидетельствует о том, что локальные флуктуации волнения не передаются в полном объеме [11]. Существенно выбиваются пики максимальных значений по данным Copernicus, что может быть связано с существенно более грубой используемой сеткой моделирования (2x2 км у Copernicus, $0,02 \times 0,02$ км у SWAN). Скаттер диаграммы измеренных и моделированных значений приведен на рис. 3.
Величины коэффициентов корреляции между измеренными значениями и данными моделирования SWAN и Copernicus составили 0,81 и 0,69 соответственно, что весьма удовлетворительно, с учетом использования данных одной метеорологической станции в качестве входных данных. Наклоны линейных трендов между измеренными и моделированными значениями находились в пределах 1-0,7, с достоверностью аппроксимации $\mathrm{R}^{2} 0,723$ и 0,632 , что демонстрирует более высокое качество работы используемой модели SWAN для моделирования ветрового волнения в сравнении с данными Copernicus. Для статистической оценки качества моделирования был рассчитан ряд статистических параметров - среднеквадратическая ошибка (СКО), средняя ошибка и средняя абсолютная ошибка (табл. 1). 
Таблица 1 и относительно локализованного, прибреж-

Статистические характеристики сравнения измеренных данных с моделированными

\begin{tabular}{|l|c|}
\hline \multicolumn{1}{|c|}{ Статистический показатель } & Значение \\
\hline СКО (Измерения/Сopernicus) & 0,54 \\
\hline СКО (Измерения/SWAN) & 0,38 \\
\hline Средняя ошибка (Измерения/Copernicus) & 0,35 \\
\hline Средняя ошибка (Измерения/SWAN) & 0,31 \\
\hline $\begin{array}{l}\text { Средняя абсолютная ошибка (Измере- } \\
\text { ния/Сореrnicus) }\end{array}$ & 0,45 \\
\hline $\begin{array}{l}\text { Средняя абсолютная ошибка (Измере- } \\
\text { ния/SWAN) }\end{array}$ & 0,29 \\
\hline
\end{tabular}

По результатам проведенных статистических тестов данные по глобальному анализу для рассматриваемой части акватории Балтийского моря имеют большее отклонение от измеренных значений $(\mathrm{CKO}=0,54)$; для анализа, основанного на WAM, это является приемлемым показателем [22; 23]. При этом полученные посредством SWAN результаты $(\mathrm{CKO}=0,38)$ сопоставимы, а также несколько выше, чем в ранее приводимых исследованиях [11; 24], что связано с использованием густой расчетной сетки ного участка моделирования. Эти факты обоснованно позволили принять результаты моделирования ветрового волнения с использованием модели SWAN в качестве гидродинамических условий для моделирования ширины затопления пляжа.

\section{Моделирование ширины затопления пляжа и сравнение полученных результатов}

На втором этапе был выполнен расчет ширины затопления пляжа на основе стандарта СП 38.13330.2018, а также по адаптированной для юго-восточной Балтики модели. Результаты моделирования были сопоставлены с данными натурных наблюдений на берегу Куршской косы (рис. 4).

По результатам сравнения ширины затопления пляжа по адаптированной модели и СП 38.13330.2018 первая показала существенное преимущество в точности определения ширины затопления пляжа. СКО составила по результатам сравнения 0,73 , при средней абсолютной ошибке определения ширины в 0,52 м. Статистические характеристики приведены в табл. 2.

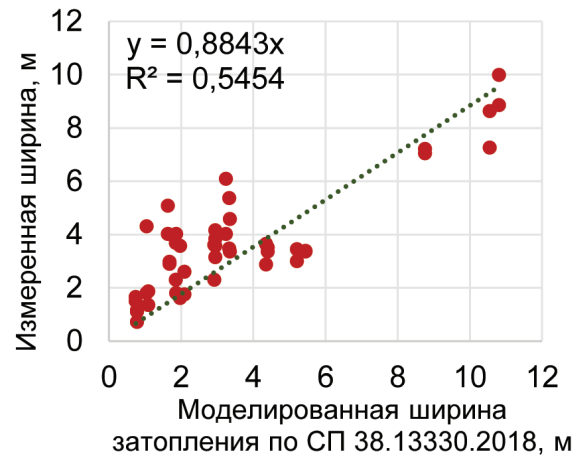

a)

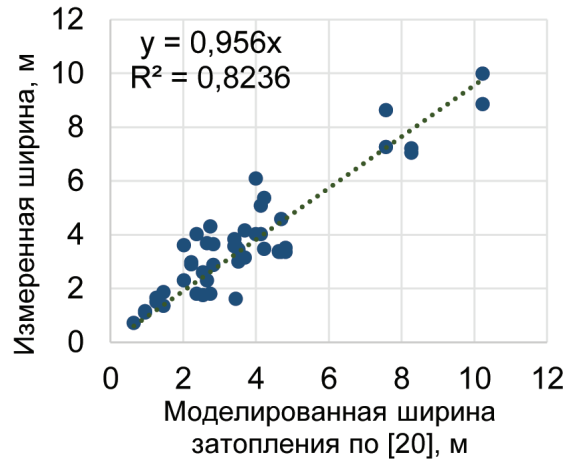

б)

Рис. 4. Скаттер диаграммы измеренных и моделированных значений: а) СП 38.13330.2018 и измеренные значения; б) [20] и измеренные значения

Таблица 2

Статистические характеристики сравнения измеренных данных с моделированными

\begin{tabular}{|l|c|}
\hline \multicolumn{1}{|c|}{ Статистический показатель } & Значение \\
\hline СКО (Измерения/СП 38.13330.2018) & 1,82 \\
\hline СКО (Измерения/[20]) & 0,73 \\
\hline Средняя ошибка (Измерения/СП 38.13330.2018) & 0,16 \\
\hline Средняя ошибка (Измерения/[20]) & 0,02 \\
\hline Средняя абсолютная ошибка (Измерения/СП 38.13330.2018) & 1,61 \\
\hline Средняя абсолютная ошибка (Измерения/[20]) & 0,52 \\
\hline
\end{tabular}


Несмотря на рекомендацию по использованию при расчете высоты наката волн использовать натурные наблюдения, которые чаще всего отсутствуют, СП 38.13330.2018 предлагает расчетную методику, которая может быть обобщена на побережье морей России. По проведенному сравнению с натурными данными локальные адаптации расчета существенно превосходят полученные при руководстве нормой результаты (СКО для СП 38.13330.2018 составила 1,82). Таким образом, при проведении научных исследований следует подбирать локальные адаптации моделей волнового наката, точнее характеризующие гидродинамический процесс $[20 ; 25]$.

\section{Заключение}

Проведенное исследование показало возможность и непосредственное сочетание моделирования ветрового волнения с использованием спектральной волновой модели с моделированием ширины затопления пляжа. По результатам сравнения с натурными данными уровнемера-волнографа на платформе Д6, данными E.U. Copernicus Marine Service результаты моделирования SWAN существенно выше, чем в глобальной модели, несмотря на использование данных одного метеопоста, установленного в открытом море. Выполненное моделирование ширины затопления пляжа с использованием национальных методик и регионально адаптированной модели волнового наката показало значительное улучшение качества определения ширины с использованием локальных моделей. При проведении исследования ширины затопления пляжа как лимитирующего фактора развития прибрежных дюн большую репрезентативность результатов можно получить при использовании региональной адаптации. Однако для большей достоверности расчетов следует производить регулярные наблюдения за состоянием пляжей, что позволит существенно улучшить качество производимых оценок. Научно обоснованные и точные оценки могут позволить принять рациональное решение по защите и восстановлению прибрежных дюн Национального парка «Куршская коса», имеющих важное экологическое и культурное значение.

Исследование выполнено при финансовой поддержке РФФИ и Калининградской области в рамках проекта № 19-45-393001. Сбор данных волнографа был выполнен при поддержке госзадания ИО РАН (тема № 0149-2019-0013).

\section{Список литературы / References}

1. Hesp P. Foredunes and blowouts: initiation, geomorphology and dynamics. Geomorphology. 2002. vol. 48. № 1-3. P. 245-268.

2. Delgado-Fernandez I. A review of the application of the fetch effect to modelling sand supply to coastal foredunes. Aeolian Research. 2010. vol. 2. № 2-3. P. 61-70.

3. Bauer B.O., Davidson-Arnott R. G. D. A general framework for modeling sediment supply to coastal dunes including wind angle, beach geometry, and fetch effects. Geomorphology. 2003. vol. 49. № 1-2. P. 89-108.

4. Danchenkov A., Belov N., Stont Z. Using the terrestrial laser scanning technique for aeolian sediment transport assessment in the coastal zone in seasonal scale. Estuarine, Coastal and Shelf Science. 2019. vol. 223. P. 105-114. DOI:10.1016/j. ecss.2019.04.044

5. Booij N., Ris R.C., Holthuijsen L.H. A third-generation wave model for coastal regions: 1 . Model description and validation. Journal of geophysical research: Oceans. 1999. vol. 104. № C4. P. 7649-7666.

6. Zijlema M. Computation of wind-wave spectra in coastal waters with SWAN on unstructured grids. Coastal Engineering. 2010. vol. 57. № 3. P. 267-277.

7. Booij N., Holthuijsen L.H., Ris R. . The «SWAN» wave model for shallow water. Coastal Engineering. 1997. Vol. 1. № 4. P. 668-676.

8. Van der Westhuysen A. Modeling nearshore wave processes. ECWMF Workshop on Ocean Waves, European Centre for medium-range weather forecasts, Reading. 2012. Vol. 1. № 1. P. 50-61.

9. Cieślikiewicz W., Dudkowska A., Gic-Grusza G., Jedrasik J. Extreme bottom velocities induced by wind wave and currents in the Gulf of Gdańsk. Ocean Dynamics. 2017. vol. 67. № 11. P. 1461-1480. DOI: 10.1007/s10236-017-1098-4.

10. Kasiulis E., Kofoed J., Povilaitis A., Radzevicius A. Spatial Distribution of the Baltic Sea Near-Shore Wave Power Potential along the Coast of Klaipeda, Lithuania. Energies. 2017. vol. 10. no. 12. P. 2170. DOI: 10.3390/en10122170.

11. Медведева А.Ю., Архипкин В.С., Мысленков С.А., Зилитинкевич С.С. Волновой климат Балтийского моря на основе результатов, полученных с помощью спектральной модели SWAN // Вестник Московского университета. Серия 5. География. 2015. № 1. С. 12-22.

Medvedeva A.Y., Arkhipkin V.S., Myslenkov S.A., Zilitinkevich S.S. Wave climate of the Baltic sea following the results of the SWAN spectral model application// Vestnik Moskovskogo universiteta. Seriya 5 Geografiya. 2015. № 1. P. 12-22 (in Russian).

12. Медведева А.Ю., Мысленков С.А., Медведев И.П., Архипкин В.С., Кречик В.А., Добролюбов С.А. Моделирование ветрового волнения в Балтийском море на прямоугольной и неструктурной сетках на основе реанализа NCEP/ CFSR // Труды Гидрометеорологического научно-исследовательского центра Российской Федерации. 2016. № 362. C. $37-54$.

Medvedeva A.Yu., Myslenkov S.A., Medvedev I.P., Arkhipkin V.S., Krechik V.A., Dobrolyubov S.A. Modeling of wind waves in the Baltic sea on rectangular and non-structural grids based on the reanalysis of NCEP/CFSR // Trudy Gidrometeorologicheskogo nauchno-issledovatel'skogo tsentra Rossiyskoy Federatsii. 2016. № 362. P. 37-54 (in Russian).

13. Myslenkov S.A., Medvedeva A.Yu. Wave energy resources of the Baltic Sea and coastal zone of the Kaliningrad Region. Fundamentalnaya i Prikladnaya Gidrofizika. 2019. vol. 12. № 2. P. 34-42. DOI: 10.7868/S2073667319020059.

14. Kutupoglu V., Cakmak R.E., Akpınar A., van Vledder G.P. Setup and evaluation of a SWAN wind wave model for the Sea of Marmara. Ocean Engineering. 2018. vol. 165. P. 450-464.

15. Akpınar A., Bingölbali B., Van Vledder G. P. Wind and wave characteristics in the Black Sea based on the SWAN wave model forced with the CFSR winds. Ocean Engineering. 2016. vol. 126. P. $276-298$. 
16. Sharp E., Dodds P., Barrett M., Spataru C. Evaluating the accuracy of CFSR reanalysis hourly wind speed forecasts for the UK, using in situ measurements and geographical information. Renewable Energy. 2015. vol. 77. P. 527-538. DOI: 10.1016/j.renene.2014.12.025.

17. Gelumbauskaite L.Z., Grigelis A., Cato I. Topography and sediment maps of the Central Baltic Sea. 1999. 20 p.

18. Komen G.J., Hasselmann K., Hasselmann K. On the existence of a fully developed wind-sea spectrum. Journal of physical oceanography. 1984. vol. 14. № 8. Р. 1271-1285.

19. СП 38.13330.2018. Нагрузки и воздействия на гидротехнические сооружения (волновые, ледовые и от судов). СНиП 2.06.04-82. М.: ОАО «ВНИИГ им. Б.Е. Веденеева», 2019, $106 \mathrm{c}$

20. Paprotny D., Andrzejewski P., Terefenko P., Furmańczyk K. Application of empirical wave run-up formulas to the Polish Baltic Sea coast. PloS one. 2014. vol. 9. № 8. P. e105437. DOI: 10.1371/journal.pone.0105437.
21. Danchenkov A.R., Belov N.S. Morphological changes in the beach-foredune system caused by a series of storms. Terrestrial laser scanning evaluation. Russ. J. Earth Sci. 2019. vol. 19. ES4003. DOI: 10.2205/2019ES000665.

22. Soomere T. Extremes and decadal variations of the northern Baltic Sea wave conditions. Springer, Dordrecht: Extreme Ocean Waves. 2008. P. 139-157. DOI: 10.1007/ 978-1-4020-8314-3_8.

23. Blomgren S., Larson M., Hanson H. Numerical modeling of the wave climate in the Southern Baltic Sea. Journal of coastal research. 2001. vol. 17. № 2. P. 342-352.

24. Saremi S. Development of a Wave Database in Coastal Areas around Sweden Using the SWAN Wave Model: Master's Thesis. Göteborg, 2010. 56 p.

25. Aniskiewicz P., Benedyczak R., Furmańczyk K., Andrzejewski P. Validation of empirical wave run-up formulas to the Polish Baltic Sea coast. Journal of Coastal Research. 2016. vol. 75. № sp1. P. 243-247. 